\title{
A New Content-Based Search Mechanism for Image Retrieval Search Engine
}

\author{
Jasmine K. S., R. V. College of Engineering, India \\ Rishav Raj, R. V. College of Engineering, India \\ Mahalakshmi Mabla Naik, R. V. College of Engineering, India
}

\begin{abstract}
In the growing world of technology, where everything is available in just one click, the user expectations have increased with time. In the era of search engines, where Google and Yahoo are providing the facility to search through text and voice and image, it has become a complex work to handle all the operations, and a lot more data storage is needed. It is also a time-consuming process. In the proposed image retrieval search engine, the user enters the queried image and that image is being matched with the template images. The proposed approach takes the input image with 15\% accuracy to $100 \%$ accuracy to retrieve the intended image by the user. But it is found that due to the efficiency of the applied algorithm, in all cases, the retrieved images are with the same accuracy irrespective of the input query image accuracy. This implementation is very useful in the fields of forensics, defense, and diagnostics systems in the medical field.
\end{abstract}

\section{KEYWORDS}

CBIR, Clipart, Content-Based Image Retrieval, Correlation Algorithm, Image Search Engine, Image-Based Diagnostic System, Medical Imaging, RGB

\section{INTRODUCTION}

In the present era of growing world of technology, the user wants the results of whatever user searches in a better and compact way. The Search Engines like Google, Bing etc. provide the facilities to search the information required using the three functionalities that are using text, using voice and also by using image. In the available Image Search Engines, when less than 50\% part of image is entered as a query it is not able to retrieve the accurate result. Thus, the content-based image retrieval search engine is necessary. For searching about a monument or person or a place, it is necessary to know what it is called so that a text query can be formed. But in a case where one only has an image of the object/monument/person, it is difficult to form the query. The design of the search engine proposed here holds a possible solution to this problem. The objectives of the developing the search engine are: -

- To create a dataset with different types of template patterns

- To detect the level of accuracy in matching the image queried by the user

There are multiple CBIR techniques developed seeing the advancement in the image which helped in displaying the accurate output back to the user as expected. The techniques are mentioned below: -

- Query techniques based CBIR Retrieval 
- Color based CBIR Retrieval

- Texture based CBIR Retrieval

- Shape based CBIR Retrieval

- Query techniques based CBIR Retrieval: In this technique of query based CBIR Retrieval, the rough image is being passed to the system and then taking that as a form of example the algorithm applied in a top down manner to find out the resultant image related to the queried image.

- Colour based CBIR Retrieval : Computing distance measure based on colour has always been a keen interest of development where the image retrieval mechanism completely focusses on the colour which is evident and unique in between multiple orientations of image. That means if the image is rotated also, the colour pixels will not change and will remain the same in order to form the picture. In this technique, all the results rely in developing the histogram, will be implemented and be a key component in order to identify and display the proper image back to the user precisely.

- Texture based CBIR Retrieval: Texture measures identifies the visual patterns in the image and how they are spatially represented. Texture based retrieval are implemented by a number of combinational sets depending upon the count of textures being detected in the image. Texture is a property which allows the user to generate information about the spatial arrangement of color or intensities in an image or selected region of an image. Texture is the key ingredient used to identify objects or regions of interest in an image. And also, to identify that whether the image be a photomicrograph, an aerial photograph, or a satellite image.

- Shape based CBIR Retrieval: This type of CBIR Retrieval technique is used in Edge detection of an image. Here, the Shape does not convey the shape of the image and it conveys the portion of image that is being sought out. This type of technique also focusses on the boundary of the image and is invariant to translation, rotation, and scale.

\section{BACKGROUND STUDY}

Current search engines crawl the Web, download content, and digest this content locally. For multimedia content, this involves considerable volumes of data. Furthermore, this process covers only publicly available content because content providers are concerned that they otherwise lose control over the distribution of their intellectual property. The availability of vast amounts of multimedia contents in the Internet requires sophisticated means for searching and retrieval. Current search engines are generally based on a centralized gatherer which traverses the hyperlinks of the World Wide Web starting from known entry points, and which retrieves and digests all relevant data found (Graceline Abigail P., Dr. Josephine Prem Kumar,2016). Content-based retrieval (CBR) research strives to create a retrieval system that utilises digital content in the indexing process in a way that is ultimately independent of manual work. CBR is an umbrella term for content-based multimedia retrieval (CBMR), content based visual information retrieval (CBVIR), content-based image retrieval (CBIR) content-based video retrieval (CBVR) and content-based audio retrieval (CBAR) (A.K. Jain, J.E. Lee, R. Jin and N. Gregg, 2009). CBR may also be referred to as multimedia information retrieval (MIR). CBR originates from the fields of pictorial databases and visual information management, where the first systems were textual database management systems with manually-created metadata and a query language for pictures. In addition to the early pictorial database development, another branch of research emerged with the focus on time-dependent information. The first studies that have been influential in the content-based analysis of temporal data were from the fields of knowledge representation and artificial intelligence. Content based Image Retrieval System has always been a diversified subject for researchers to research upon. Multiple branches of CBIR are being implemented at many places in the industry and are working as perfect as possible and helping user to get to the best possible precise result. Like Google behind the Google Search Engine uses multiple algorithms to generate results for the user in those multiple algorithms one is Content based Image Retrieval. In 
one of the papers the author focusses on the five key modules of the general framework, i.e., query formation, image representation, image indexing, retrieval scoring, and search re-ranking. The author also focusses on finding the key problems and categorized a variety of representative strategies and methods and after that the author has summarised the complete research with 8 modules that will boost the advance of content-based image retrieval in the near future (Wengang Zhou, Houqiang Li, and Qi Tian Fellow,2017). The author of the other paper describes the main objective of image mining and also discusses how CBIR can be implemented in order to identify and display the Satellite images with different resolutions of the image. The author uses the JSEG algorithm (which is for First images are segmented into several parts) which efficiently segment the multispectral thematic map images to obtain the region level description and region level description of the image is more comprehensible for users. The image used here in this proposed methodology is Satellite images. The implemented methodology opted is for Satellite images and is specially for natural scenes other than satellite images (Shubha.G. Sanu1, Pushpa.S. Tamase,2017). The Author of this paper implements the Log based CBIR System which is used in digital image processing. In this paper several feature modification and subspace learning based relevance feedback methods are also been evaluated. The methodology implemented focusses on the local information of image as it can play an important role while creating difference between the similar images and dissimilar images (

Athira Mohanan1and Sabitha Raju,2017). The Author in this paper goes through a detailed study about CBIR and also focusses on the availability of image features like shape, colour distribution and many other factors. The paper also proposes the working of basic query by image technique which will make the process of image retrieval more promising (Shailesh Kumar and Arun Kumar Shukla,2017). The Author in this paper has presented two novel methods to enhance the retrieval performance of image retrieval system. The first approach defines the most efficient GLCM texture feature for retrieval process and the other methodology combines the colour and texture feature to improve the retrieval performance using HSV Colour Histogram (Hany F. Atlam, Gamal Attiya,2017). In this paper, the author discusses various approaches and recent evolutions that have a impact on the usage of content based image retrieval process where the various properties of CBIR is utilized that can be used for extraction of features (Parul Nangia, Sonika Jindal ,2017) .In this paper, Author's main aim is to present a simple and efficient approach for searching and retrieving the lung CT images from the large medical image dataset. The implemented methodology uses the combined approach of hierarchical and k-means clustering to provide the precise and proficient image retrieval system and computes the efficient result than other clustering techniques (Neha Malviya, Dr. Naveen Choudhary, Kalpana Jain, 2017).

\section{DIFFERENT IMAGE RETRIEVAL TECHNIQUES}

There are many techniques available for Image Processing like CBIR (Content Based Image Retrieval, TBIR (Text Based Image Retrieval), OBIR (Object Based Image Retrieval) available for matching the image with the image provided by the user and there are many algorithms also to implement this process. In an image retrieval system, several components are needed. A segmentation component is needed if one wants to retrieve an object that is only a part of Image. The images in a database are segmented and the segments (the interesting parts of the images) can be stored in an index structure. This structure is used to avoid the linear behaviour of the search process. An important component is also an image editor which can be used to create or edit a query image.

\section{CBIR (Content Based Image Retrieval Search Engine)}

In this technique of image processing of image queried by user is done using Image Distance. An image distance measure compares the similarity of two images in various dimensions such as color, texture, shape, and others. In this technique, the algorithm works like when the user queries 
Table 1. Comparison of CBIR technique and TBR technique

\begin{tabular}{|l|l|l|}
\hline Parameters & CBIR technique & TBIR technique \\
\hline Full Form & Content based Image Retrieval & Text based Image Retrieval \\
\hline Query based on & Image & Text \\
\hline Procedure to produce result & $\begin{array}{l}\text { Completely based on image queried } \\
\text { by the user }\end{array}$ & $\begin{array}{l}\text { Based on the keywords surrounding } \\
\text { images, and that are used to describe } \\
\text { image content. }\end{array}$ \\
\hline Search on the basis of & $\begin{array}{l}\text { using the text to describe the } \\
\text { name, the content, the size and the } \\
\text { compression method of the image }\end{array}$ & $\begin{array}{l}\text { Using the visual features such as text, } \\
\text { colour, shape, texture and their spatial } \\
\text { relations }\end{array}$ \\
\hline
\end{tabular}

for an image from the proposed system, the algorithm takes the image as input and then processes it on the basis of the image given by the user, but not by the content written over it.

\section{ALGORITHM IMPLEMENTED}

"Correlation Algorithm" is a kind of algorithm highly used in image processing by computer vision. Image is basically a visual representation of something. It is a collection of pixels. Correlation helps the developer to understand the dependency between two variables. If they are highly correlated, it can help to build linear model and predict one variable using another. So, it is basically used to check goodness of fit.

\section{METHODOLOGY ADOPTED FOR THE PROPOSED SYSTEM}

The proposed system allows user to get the accurate result on the basis of queried image. The Methodology has the following approach:

The GUI allows user to query for image in the system and then on the basis of that the queried image is being converted into grayscale and then it goes on to template matching(here the whole dataset is also converted into Grayscale) and then the queried image along with dataset is matched and then the result is displayed back to the user.

\section{BLOCK DIAGRAM OF THE PROPOSED SYSTEM}

The block diagram of the proposed system which will implement the Content Based approach to Image Search where the image queried image will be given as input and the matched image will be displayed to the user after processing.

Here in the proposed system the user queries for an image to the system, and that image is being converted into Grayscale and then with the help of Algorithm called "Correlation Algorithm" the system compares the images templates with the queried image and then the result is being displayed in the screen. At the comparator stage, the Algorithm comes into picture for accurate image matching.

\section{CODE SNIPPET FOR THE SYSTEM}

The Table 2 displays a code snippet which explains about the ISE (Image Search Engine) Algorithm specially designed in order to match the image with the templates with the tolerance whose pSmall value should be between margins. For this the queried image and template images need to be converted 
Figure 1. Process flow of the proposed system

\section{Input of Queried Image}

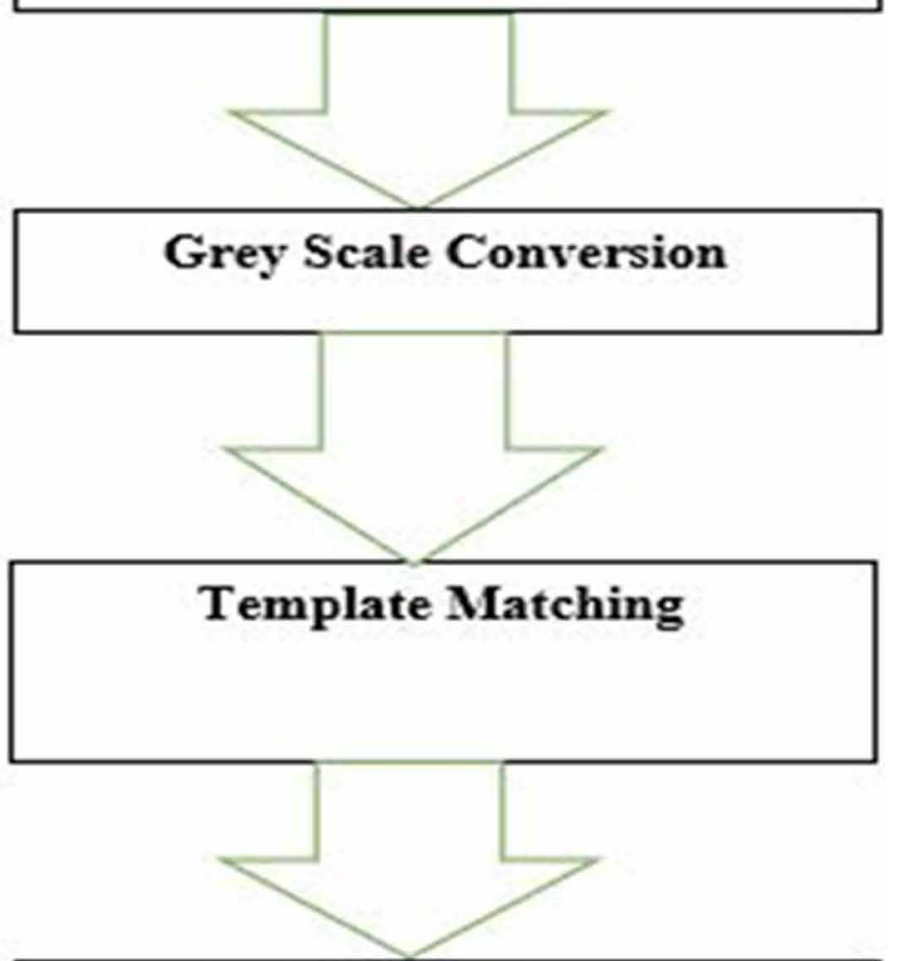

\section{Extract the similar image among all the templates}

Displaying the Queried Image

into Bitmap format and then creating a pointer of it and checking the image with the help of the algorithm.

The algorithm displayed over in the Table 3 explains about the procedure opted in order to generate the matched image and also to fetch the location of the matched Image and if there is no match found 


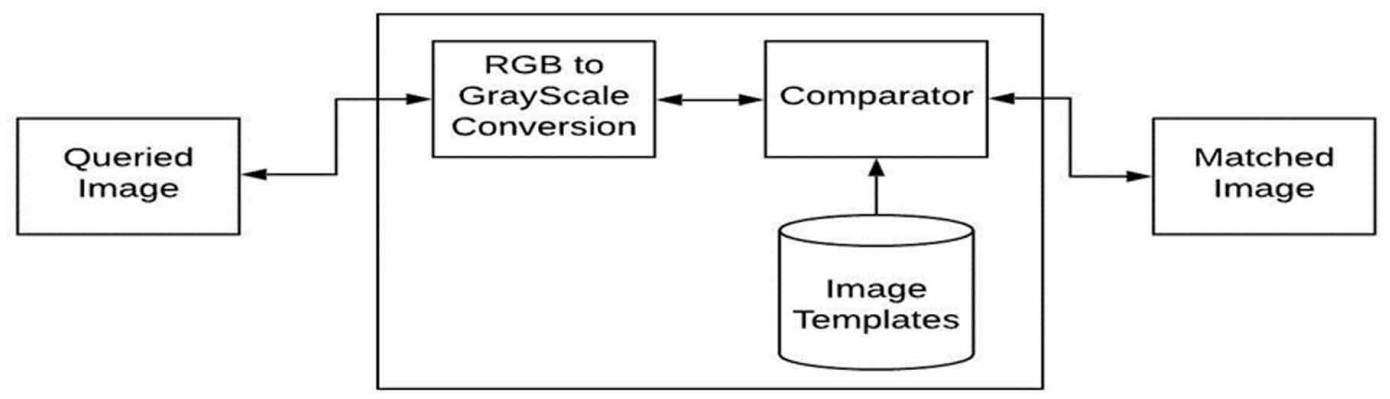

situation then the pointer will continue to iterate for other images in the template in order to find the corrected image as queried by the user.

Algorithm 3 conveys that Image height and width is also an important factor that has been considered in the Image Processing technique opted in order to reach out for the best solution as possible with accuracy. So, the algorithm designed converts the queried image into the bitmap format and then using the built-in functions and then checking the height and width of the queried image with the image available in templates.

Table 2. Algorithm for finding the Image with tolerance

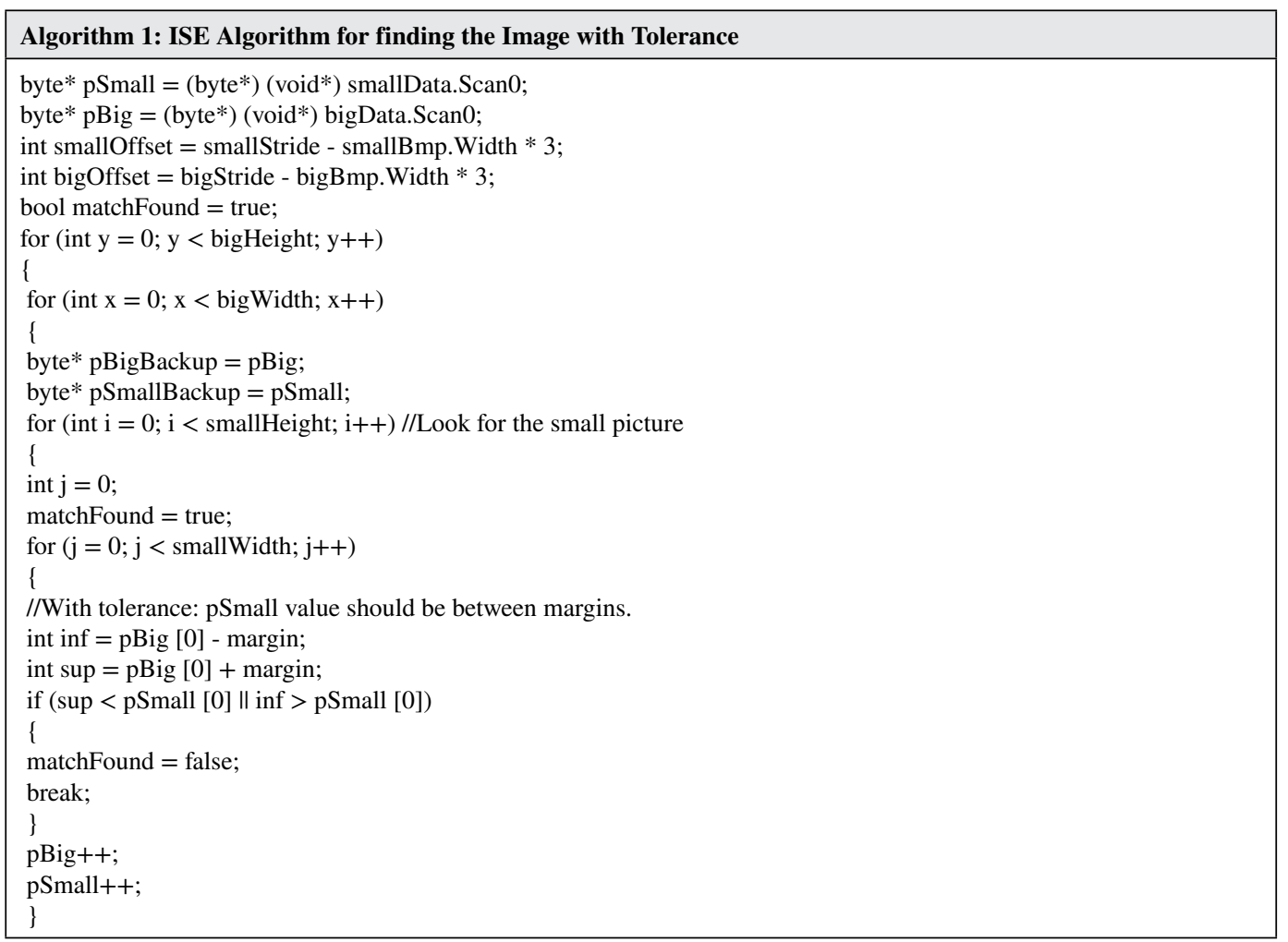


Table 3. Match generation algorithm

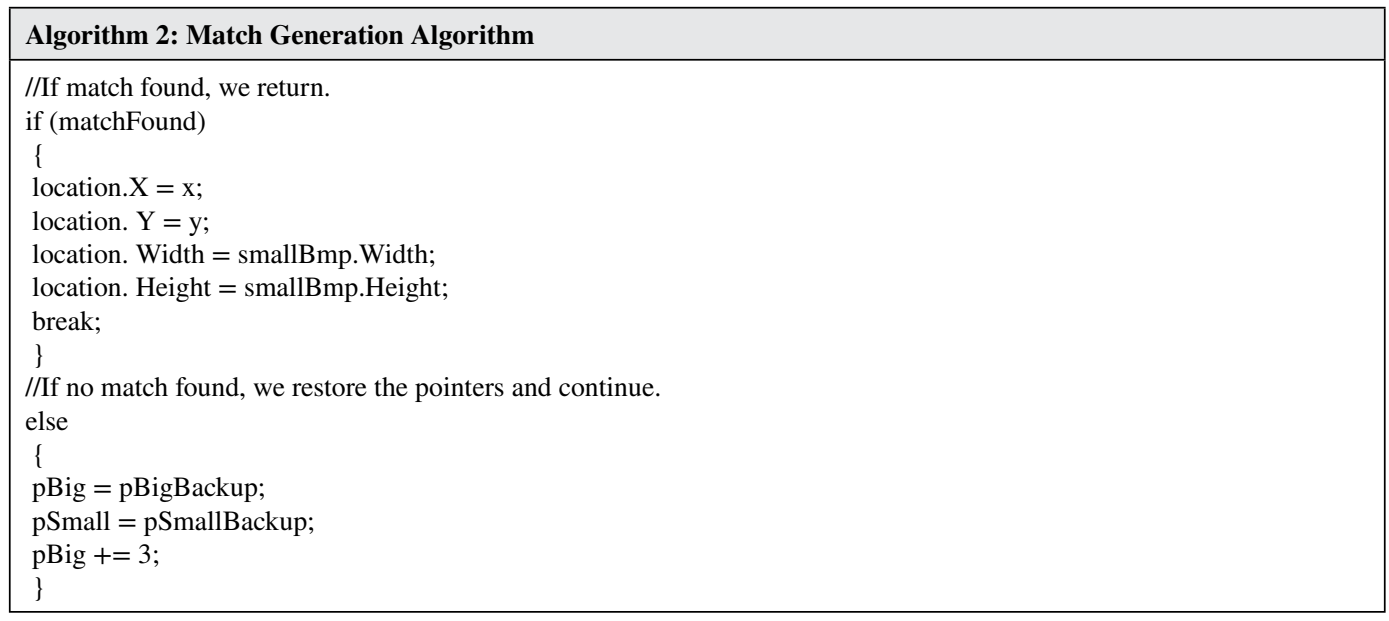

Table 4. Algorithm to match the height and width of the queried image with templates

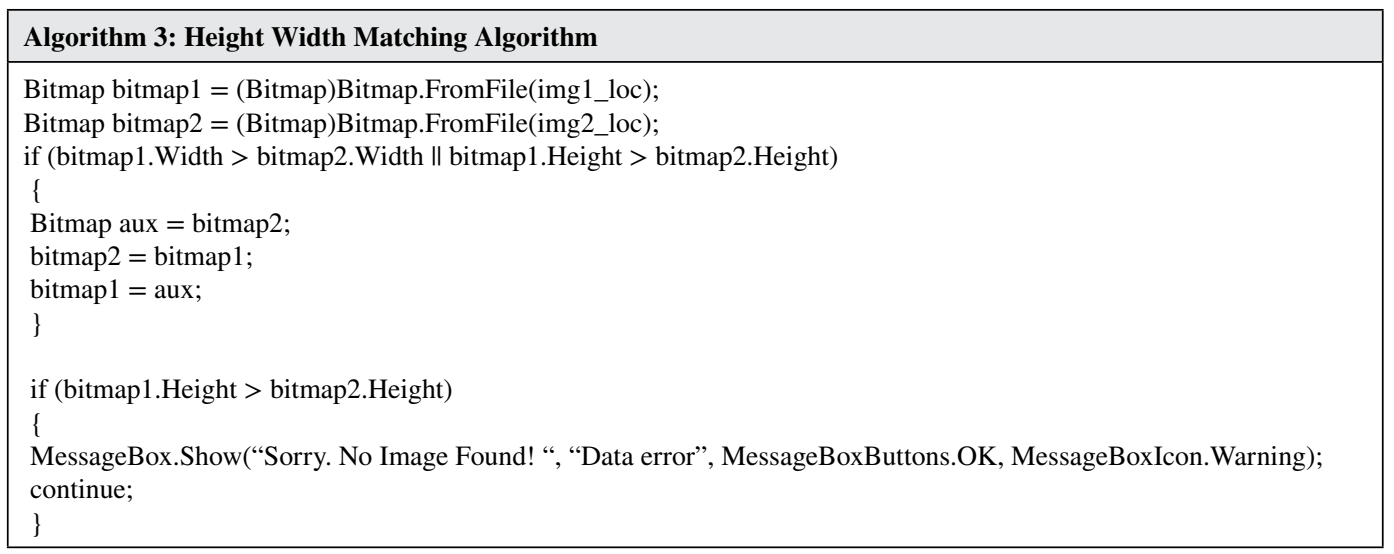

\section{INTERFACE OF THE PROPOSED SYSTEM - SCREEN SHOTS}

The Home Screen is, where the user will interact with system and allows user to enter the part of image for querying to the system in the above provided space and then click on Search Button and then the process starts for processing the image using Algorithm.

Validation also being included in the system where the user clicks on "Search" button before entering the image for querying. The error message like that which is being displayed in the Screenshot above will be given back to user with a proper message.

The above displayed screen is the output screen where the user enters part of image and the matched image is being displayed back to the user completely using CBIR technique and Correlation Algorithm. 
Figure 3. The home screen

\section{Search Image}

\section{Search Image}

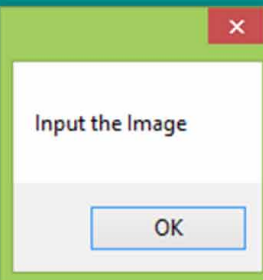

\section{CATEGORISATION OF RESULT ANALYSIS}

The Analysis of result happens in three categories as mentioned below:

- Grayscale

- colour 


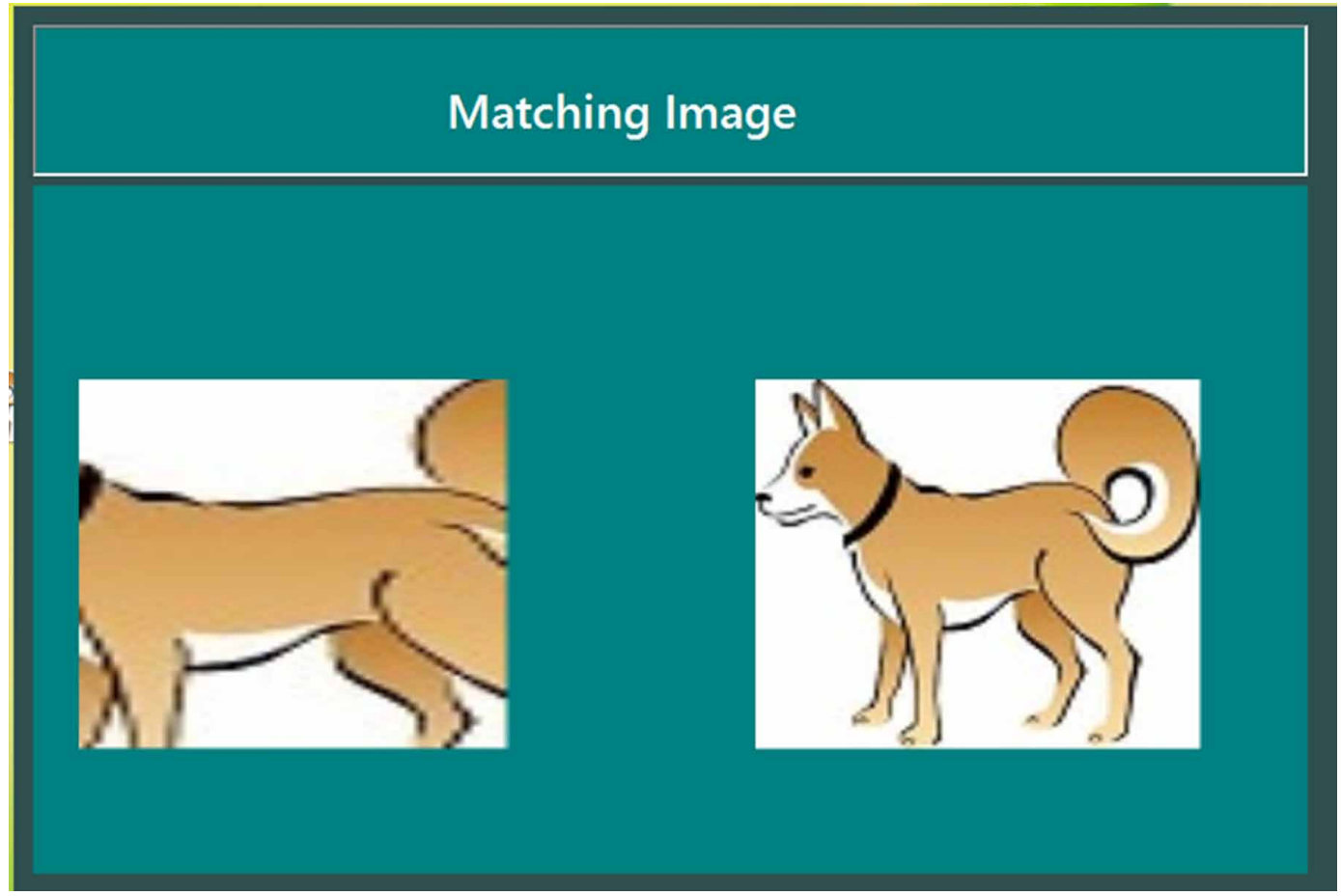

- $\%$ (Percentage) of image considered as queried image

- Grayscale : In order to start with Grayscale approach , the image is being converted into grayscale format using the built in function, Bitmap and then stored the queried image in the MATRIX format in order to implement the CBIR technique as it focusses on the content of the Image.

Figure 6 represents the form that accept the user's input image in order to find the grayscale format of the image in order to go further with the processing.

Figure 7 holds the primary step which is implemented in order to generate the Grayscale conversion of the image for the further processing.

- Colour: There are sub categories in this analysis method as to check if the user enters the image which is completely mixed with pixels like the earlier stage where the user enters the grayscale image the output is being displayed and it has also shown that accuracy is not been compromised at each and every step of implementation of the system.

- \%(Percentage) of image considered as queried image: There are multiple search engines available in the arena which proves their accuracy by implementing n- number of algorithms in order to focus on multiple parts of image because all play an important role in displaying the correct image with accuracy. In the technique called CBIR(Content Based image Retrieval) is one of the most prominent technique in image processing and in CBIR there is one algorithm which has established its position at some level and that is Correlation Algorithm which works on the image root part .Here in this category there are some sub categories which are important to be discussed like how system behaved when different percentage of image passed through the system.

- $50 \%$ of the Image 


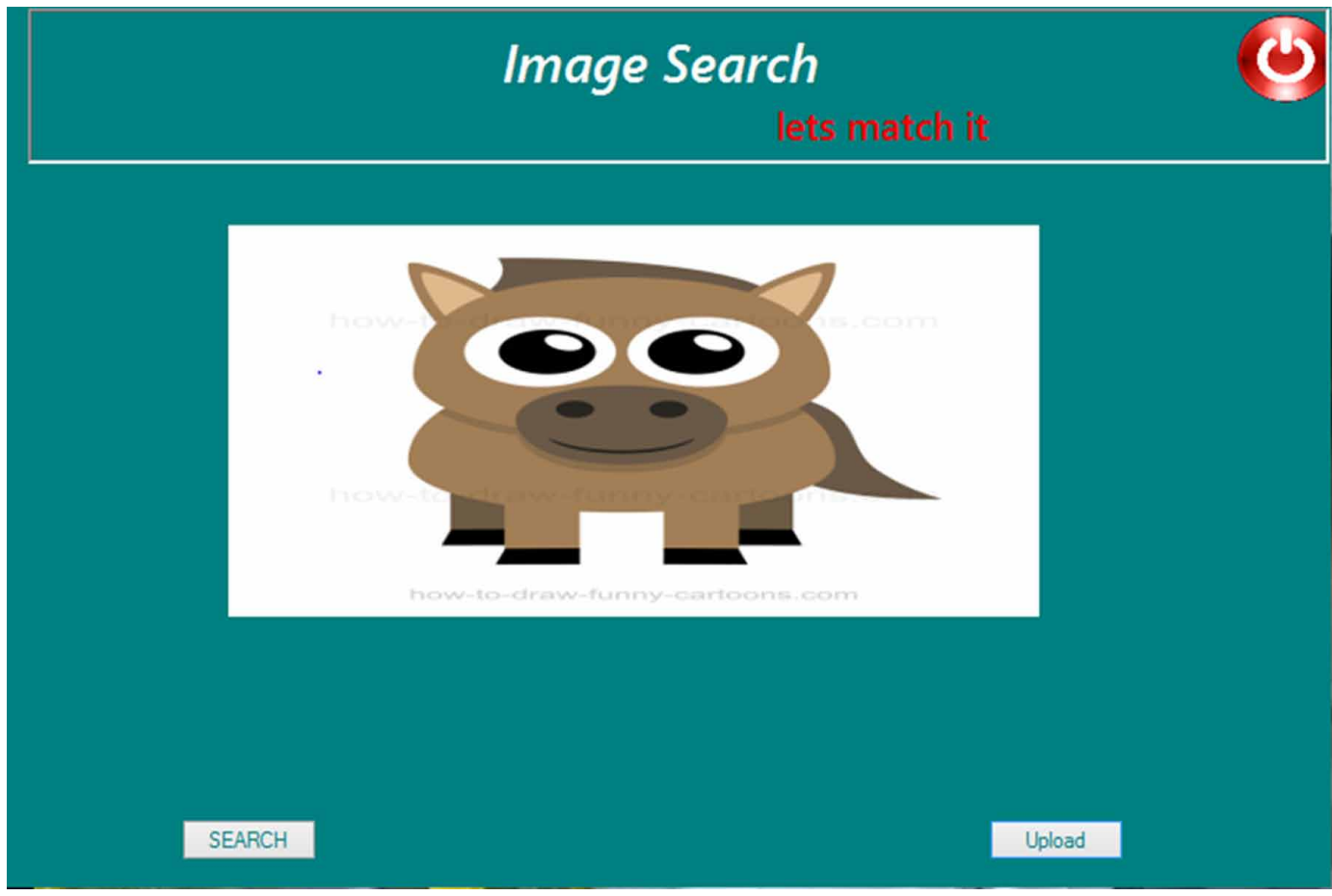

- $\quad 30 \%$ of the Image

- $15 \%$ of the Image

- $\quad \mathbf{5 0 \%}$ of the Image: The below displayed output will clarify the situation when $50 \%$ of the Image is being passed through the system as an input and the system is able to behave perfectly fine in these situations like the other Search Engines do.

Figure 8 displays the screen in which user queries for the desired output by giving input to the System.

Figure 9 displays the output generated by the developed system with accuracy when the $50 \%$ of the image is given as an input to the System.

- $\quad 30 \%$ of Image: The below displayed output will clarify the situation when $30 \%$ of the Image is being passed through the system as an input and to check whether the system is able to behave perfectly fine in these situations.

Figure 10 displays the screen in which user queries for the desired output by giving input to the System.

Figure 11 displays the output generated by the developed system with accuracy when the $30 \%$ of the image is given as an input to the System.

- $\quad$ 15\% of the Image: The below displayed output will clarify the situation when $15 \%$ of the Image is being passed through the system as an input and to check whether the system is able to behave perfectly fine in these situations. It can also be seen here the flexibility possessed by the system when the input image is decreased up to $15 \%$ of the total image (total image is the image available 


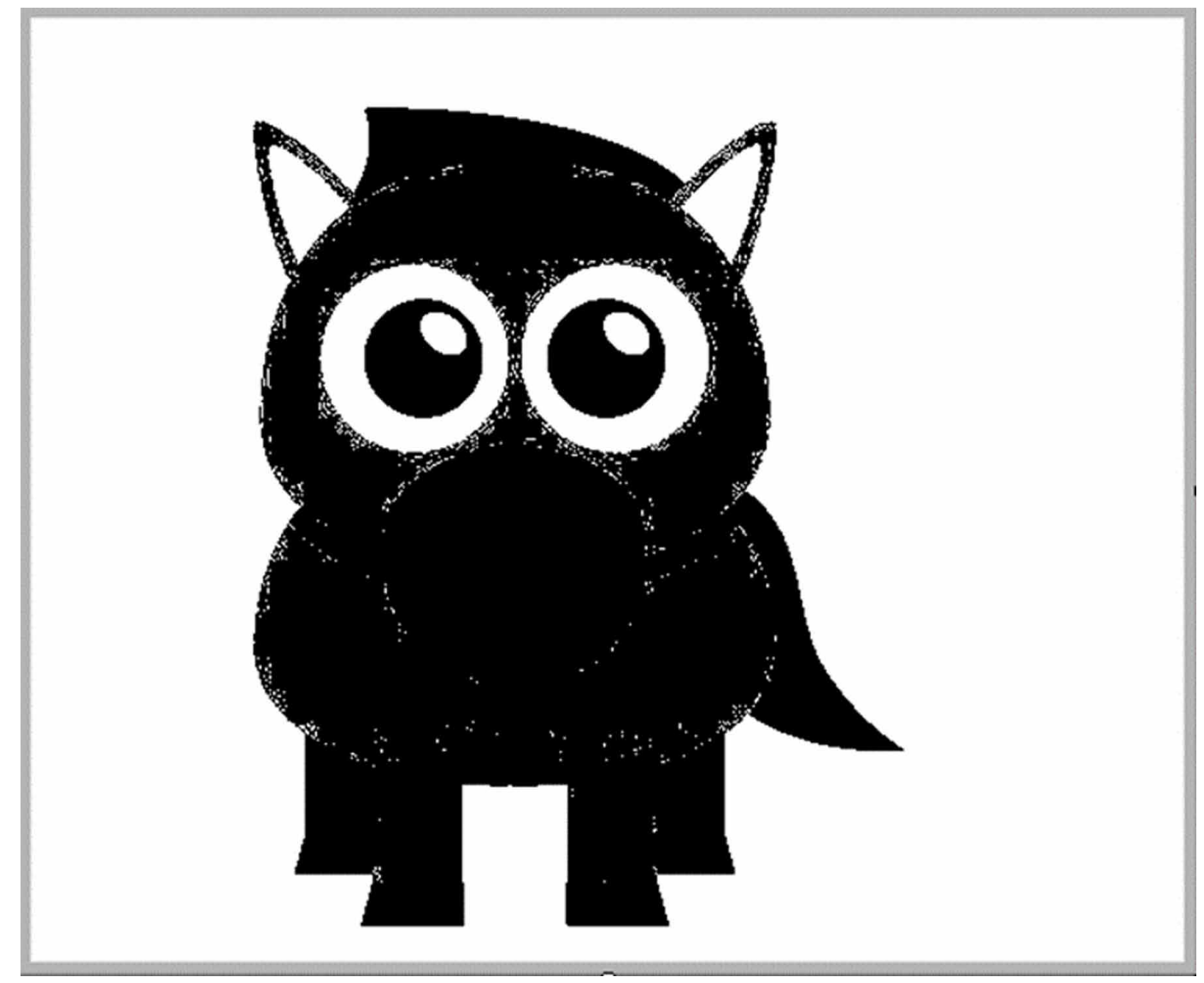

in the database).This system does not restrict user to input this much $\%$ of image as input ,the user using the system has a flexibility to input any type and \% of image into the system and system will behave properly and will display the desired image as expected by the user.

Figure 12 displays the screen in which user queries for the desired output by giving input to the System.

Figure 13 displays the output generated by the developed system with accuracy when the $15 \%$ of the image is given as an input to the System

\section{DESIGN AND IMPLEMENTATION ISSUES}

The Proposed system is being implemented in Visual Studio using C\#.Net and the database used to store the templates of different kind of images is the Windows file Directory. And the dataset used in this system is the CLIPART images, and the size of image is fixed. These are the limitations for the system where it restricts user from giving different format of images with higher contrast and image complexion like .jpeg,.png and many more. In implementation phase, it is observed that Visual Studio also cannot be implemented on server. And also, the dataset is not secure in the local system. 


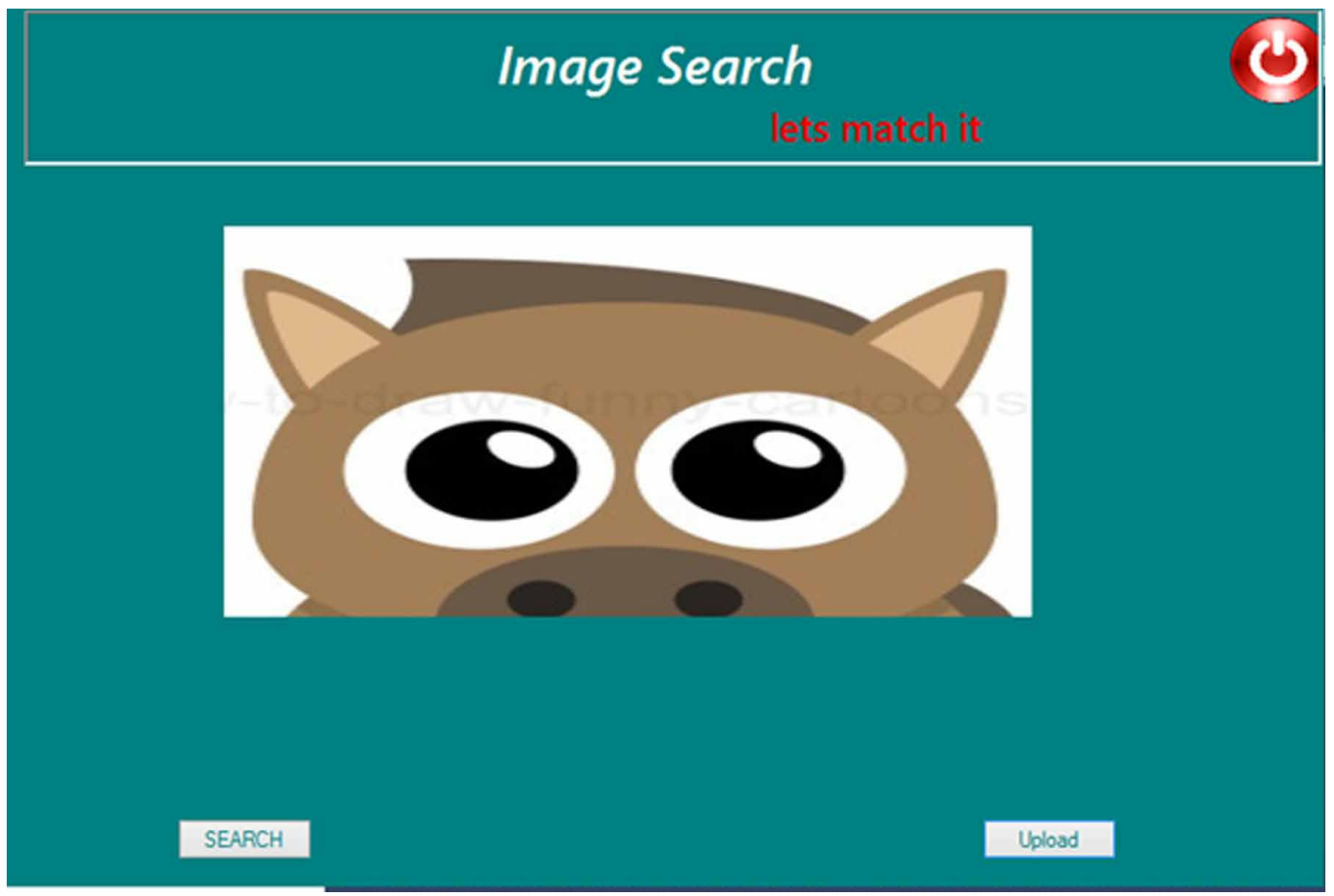

Figure 9. Generation of Output given input with $50 \%$

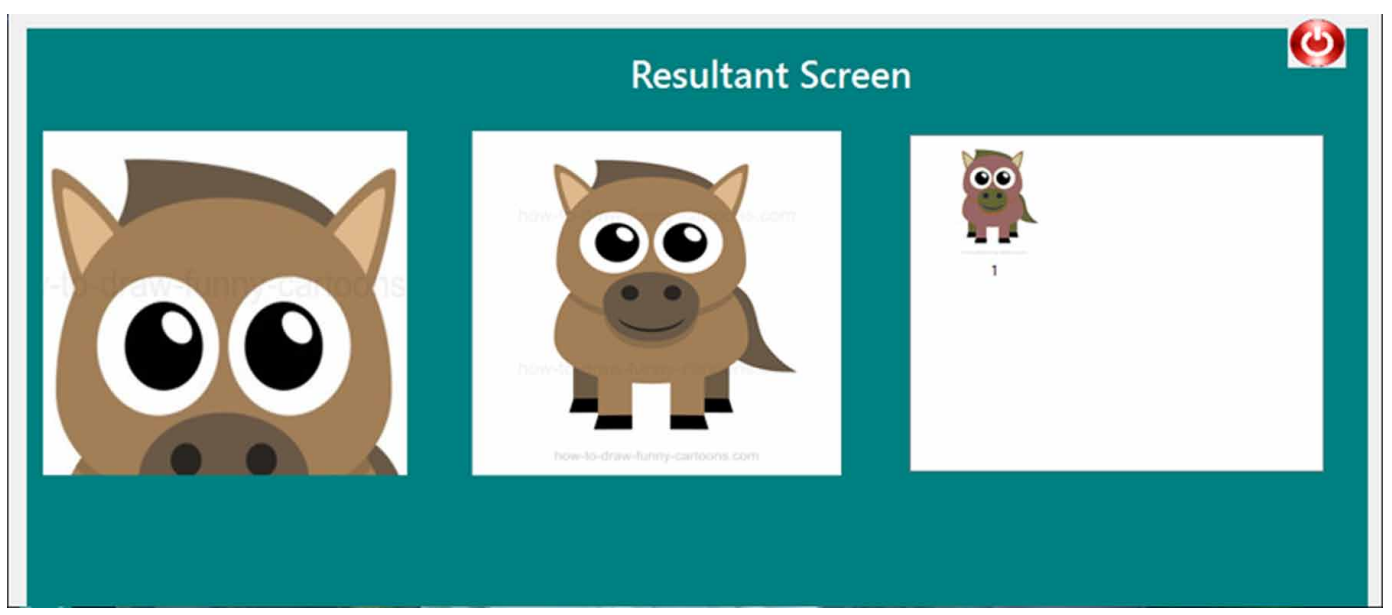

\section{NEED FOR IMAGE SEARCH ENGINE}

The Arena where we are still in, is the arena where the customers require the result of whatever been requested from the system instantly and accurately. The Image Search Engine allows user to search for the images on the basis of image queried through the developed system. The image size may be of any percentage. The Need for the image search engine is because of the technology paradigm shift where the user wants to result for the queries about anything user searches and in need of immediate and accurate response . 
Figure 10. User Queried image which is $30 \%$ of the complete Image

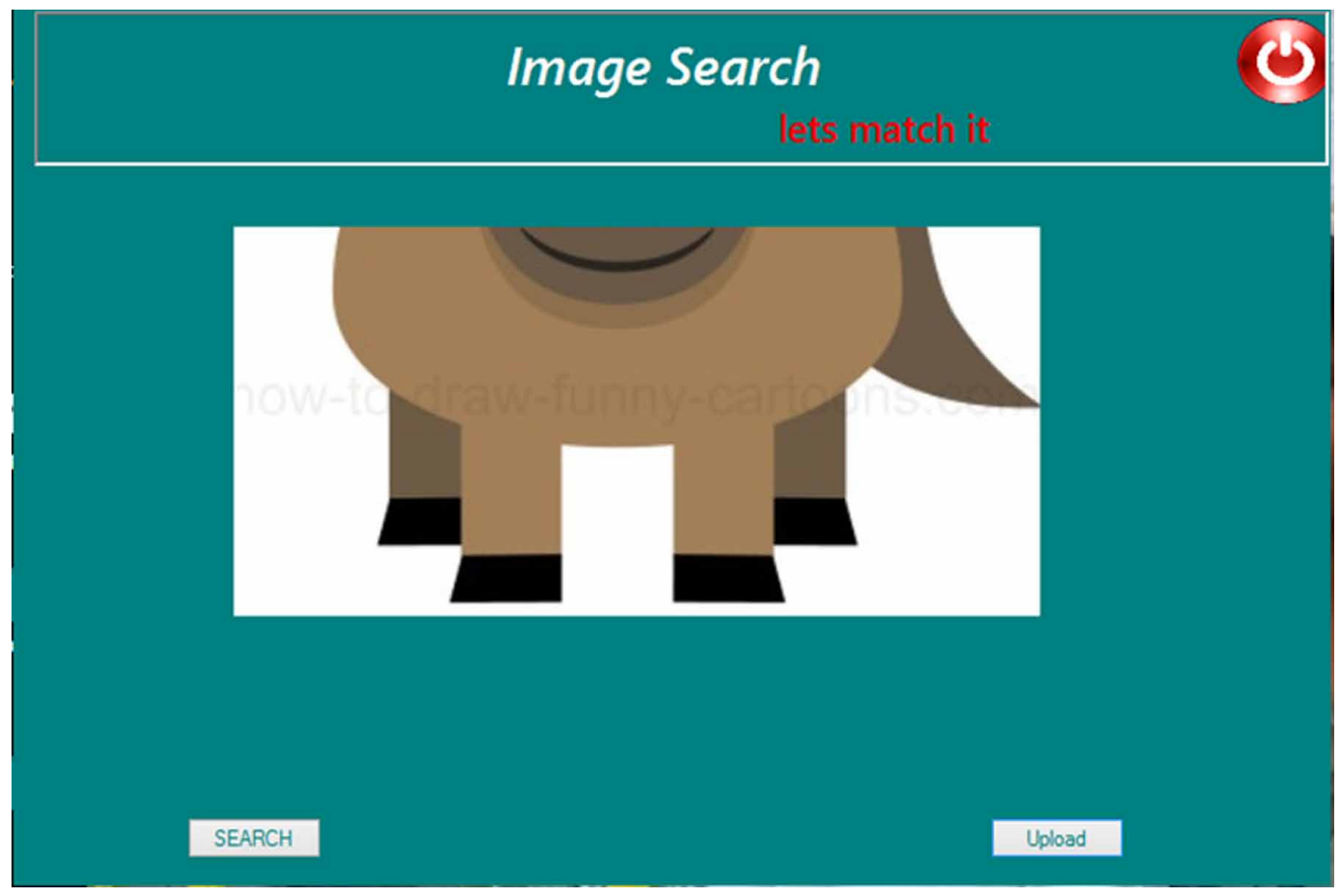

Figure 11. Generation of Output given input with $30 \%$

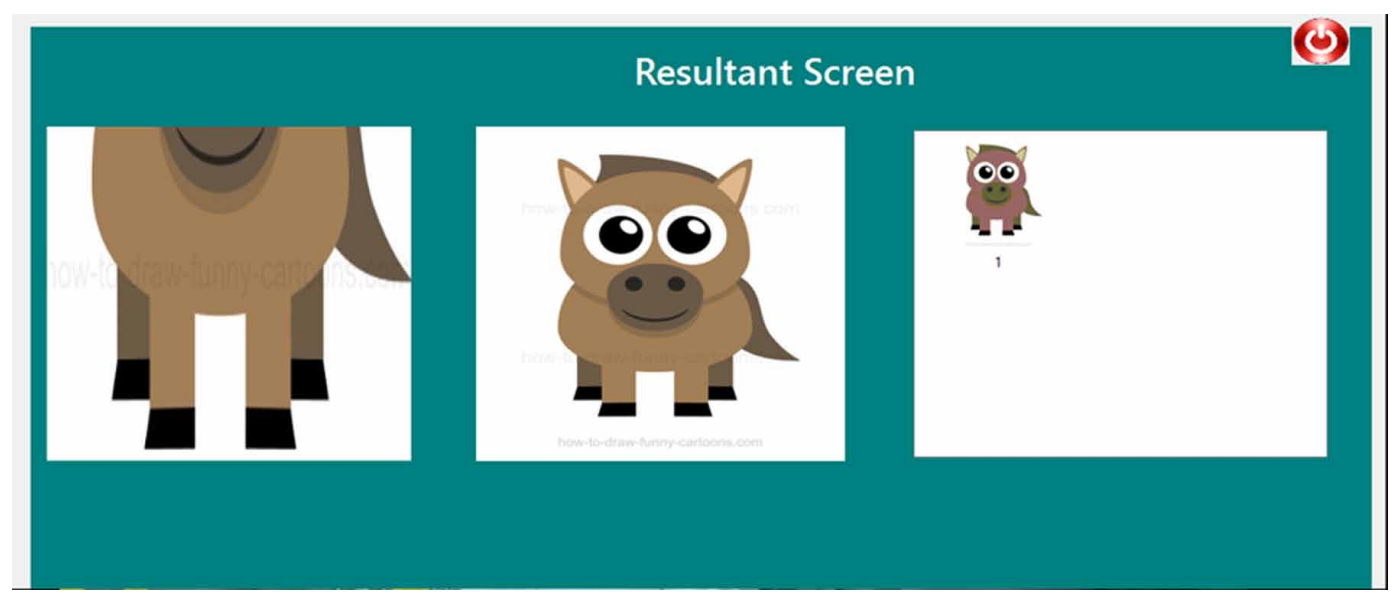

\section{CONTENT BASED IMAGE RETRIEVAL AND FEATURE EXTRACTION}

The Content Based Image Retrieval technique is one of the most effective and core techniques used by most of Image Search Engines for displaying the accurate results back to the user as per requirement. The Technique works on the core part of the image that is the content of the image and not of the background of the image because if one consider the background also at the first step of image matching process with the template, it will be more tedious task as there are multiple image 


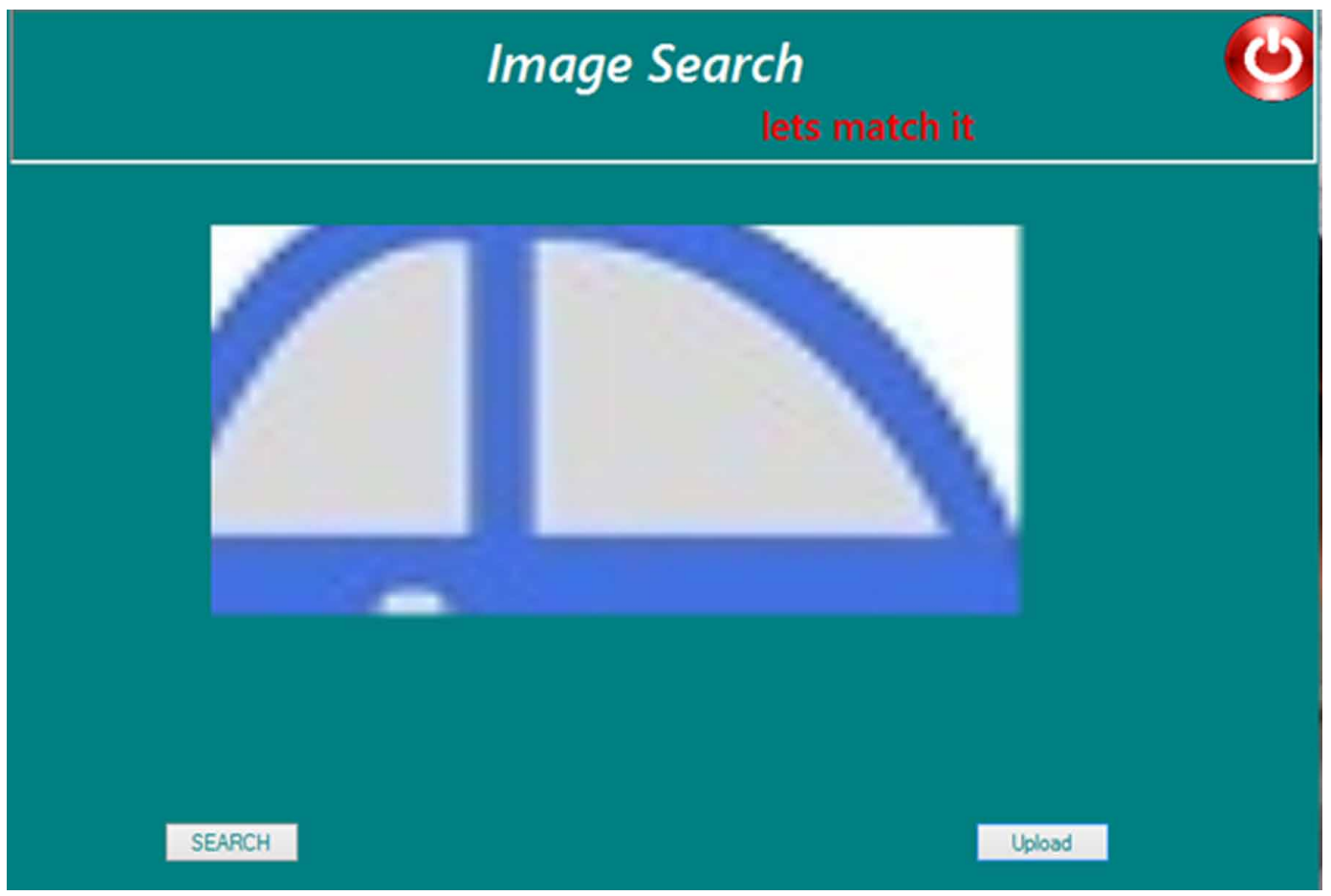

Figure 13. Generation of Output given input with $15 \%$

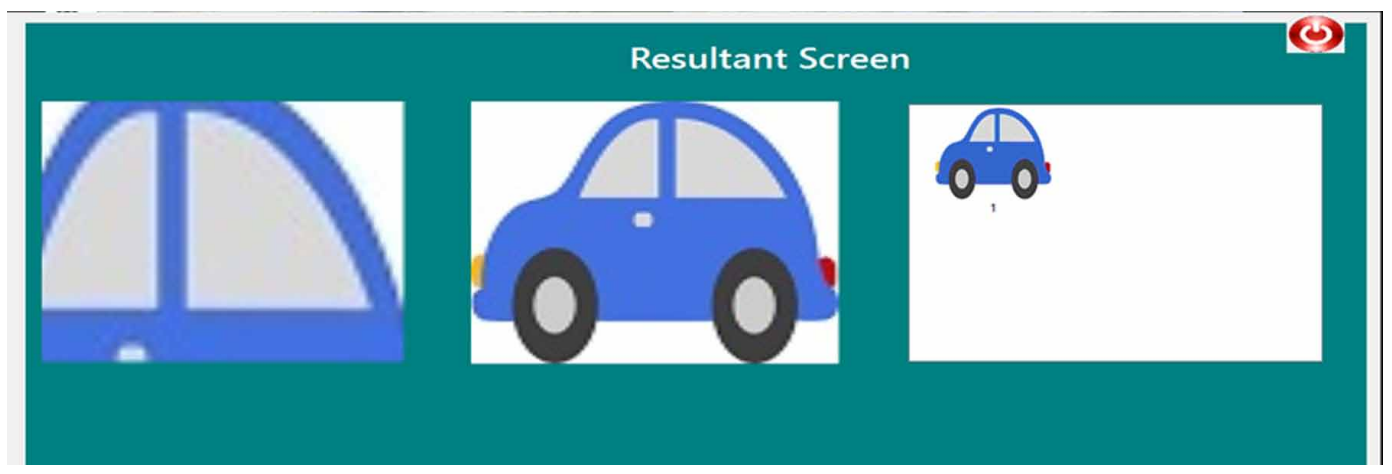

that will be clicked in that particular background and that will generate ambiguous result that is not user has queried for. To remove such situations, Content-based Image Retrieval Technique is more effective as it completely emphases on image and compares the queried image pixels with the complete templates available in the backend (Database). When the image is being passed through to the system ,it converts the image into grayscale format and then the matrix(Binary) format will be generated and then the complete template will be converted into the same matrix format and the queried image matrix is being compared with the template matrix . Using the Correlation Algorithm the matrix is being compared (each pixel will be compared sequentially) and at the end of this process the expected output will be produced on the screen with the required precision. 
The System is developed to reduce the percentage of image queried by the user as compared to the image queried by available Search Engines and the minimum accurate percentage of input image expected by the developed system is $\mathbf{1 5 \%}$ of the complete image in order to generate the accurate output because the situation will not be the same every time that the user will have at least $50 \%$ of the image. In those situations, this system is more valuable to fulfil the user requirement with precise result.

The System is best suited for the images that are of clipart kind of(as there are multiple types of image are available like .jpg,.png, and many more ). These CLIPART image are not of the complex kind of image here complex means that image will not have multiple pixels blend together to add some effects to the image. The Image sized fixed while developing this system is fixed up to $1 \mathrm{MB}$ as the image does not have multiple pixels into it. The maximum size on which the system will behave properly is up to $2 \mathrm{MB}$. The most important fact that needs to be highlighted here is that the size of image is calculated on the basis of the complexity of the image that is multiplying the surface area of a document (height $\mathrm{x}$ width) to be scanned by the bit depth and the dpi (Dots per inch).

The algorithm used in to develop this system is the Correlation algorithm which is based on sequentially matching each and every pixel of the image with the template image in order to generate the accurate result. With many advantages like accuracy and many more, the algorithm also have multiple limitations too like its take lot of time in order to generate the result and apart from that the main disadvantage of the all is that this algorithm is too simple to deal with some complex image matching problem. If the objects in two images are not exactly same, such as different orientation, different size, etc, the correlation algorithm can't give us a perfect matching result.

\section{CONCLUSION AND FUTURE WORK}

Content-based Search Engine is a technique used in the Image Search Engines to linearly check for images among the available template images. As the growing world of technology, this technique also finds its place in putting an impact in displaying the image which is being queried by the user. It also enhances the image processing techniques and algorithms do the work easier for processing the image.

The research work has its own limitations like the software is platform dependent hence it can run only on windows. The Queried image should be of jpg type but when the size reduces to very less, accuracy decreases a bit as there will be many images with the same RGB values being matched with the template images. The system can be operable by a single user at a time. It can only be imported to the system having Visual Studio 2015 installed in it.

Future Enhancements of this research includes, Remote Access, Web Implementation, Database to be added to store the images. Adding more constraints for doing image searches like Contrast, Rotation, Flip etc. The Image can be of any format like .png and the processing will be the same as per the .jpeg image processing. Some more algorithms to match image based on other parameters can also be implemented. 


\section{REFERENCES}

Atlam, Attiya, \& El-Fishawy. (2017). Integration of Color and Texture Features in CBIR System. International Journal of Computer Applications, 164(3).

Graceline \& Kumar. (2016). Study of Content Based Image Retrieval Techniques. International Journal of Advanced Research in Computer Science and Software Engineering, 6(2).

Gupta \& Jindal. (2017). Content based image retrieval using enhanced local tetra patterns. International Journal of Innovative Research in Science and Engineering, 3(1).

Gurusamy \& Nandhini. (2017). Content Based Image Retrieval using Feature Selection Techniques. International Journal for Scientific Research \& Development, 5(5).

Jain, A. K., Lee, J. E., Jin, R., \& Gregg, N. (2009). Content based image retrieval: an Application to tattoo images. IEEE International Conference on Image Processing, 2745-2748. doi:10.1109/ICIP.2009.5414140

Jain, S., \& Dhar, J. (2017). Image Based Search Engine Using Deep Learning. Proceedings of 2017 Tenth International Conference on Contemporary Computing (IC3). doi:10.1109/IC3.2017.8284301

Jaworska, T. (2016). Image Based Search Engine Using Deep Learning. SAI Computing Conference 2016, London, UK.

Khokhar \& Verma. (2017). Content Based Image Retrieval with Multi-Feature Classification by Back-propagation Neural Network. International Journal of Computer Applications Technology and Research, 6(7), 278-284.

Kumar \& Shukla. (2017). Design and Analysis of CBIR System using Hybrid PSO and K-Mean Clustering Methods. International Journal of Current Engineering and Technology, 7(2).

Mohanan \& Raju. (2017). A Survey on Different Relevance Feedback Techniques in Content Based Image Retrieval. International Research Journal of Engineering and Technology, 4(2).

Nangia, P., \& Jindal, S. (2017). A review on various approaches of CBIR using color and shape based features. International Conference on Academic research in Engineering and Management. www.conferenceworld.com

Phalke, T. N., \& Patil, A. A. (2017). Content based image retrieval using color and texture. 7th International Conference on Recent Trends in Engineering, Science \& management, Genba Sopanrao Moze College of Engineering, Science \&Management (ICRTESM-17).

Purbey, A., Sharma, M., \& Bohra, B. (2017). Review on: Content Based Image Retrieval. International Journal of Scientific \& Engineering Research, 8(1).

Sanu \& Tamase. (2017). Satellite Image Mining using Content Based Image Retrieval. IJESE, 7.

Zhou, Li, \& Tian. (n.d.). Recent Advance in Content-based Image Retrieval: A Literature Survey. IEEE. doi:10.1109/IC3.2017.8284301 